This item was submitted to Loughborough's Research Repository by the author.

Items in Figshare are protected by copyright, with all rights reserved, unless otherwise indicated.

\title{
Assessing physiotherapists' communication skills for promoting patient autonomy for self-management: reliability and validity of the communication evaluation in rehabilitation tool
}

\section{PLEASE CITE THE PUBLISHED VERSION}

https://doi.org/10.1080/09638288.2018.1443159

\section{PUBLISHER}

Taylor \& Francis

\section{VERSION}

AM (Accepted Manuscript)

\section{PUBLISHER STATEMENT}

This work is made available according to the conditions of the Creative Commons Attribution-NonCommercialNoDerivatives 4.0 International (CC BY-NC-ND 4.0) licence. Full details of this licence are available at: https://creativecommons.org/licenses/by-nc-nd/4.0/

\section{LICENCE}

CC BY-NC-ND 4.0

\section{REPOSITORY RECORD}

Murray, Aileen, Amanda M. Hall, Geoffrey C. Williams, Suzanne M. McDonough, Nikos Ntoumanis, lan Taylor, Ben Jackson, Bethan Copsey, Deirdre A. Hurley, and James Matthews. 2019. "Assessing Physiotherapists' Communication Skills for Promoting Patient Autonomy for Self-management: Reliability and Validity of the Communication Evaluation in Rehabilitation Tool”. figshare. https://hdl.handle.net/2134/32219. 


\section{Assessing physiotherapists' communication skills for promoting patient autonomy for self-management: reliability and validity of the Communication Evaluation in Rehabilitation Tool.}

Aileen Murray ${ }^{1}$, Amanda Hall ${ }^{2}$, Geoffrey C Williams ${ }^{3}$, Suzanne M McDonough ${ }^{4}$, Nikos Ntoumanis $^{5}$, Ian Taylor ${ }^{6}$, Ben Jackson ${ }^{7}$, Bethan Copsey ${ }^{8}$, Deirdre A Hurley ${ }^{1}$, James Matthews ${ }^{1}$

${ }^{1}$ School of Public Health, Physiotherapy and Sports Science, University College Dublin, Dublin, Ireland ${ }^{2}$ Faculty of Medicine, Memorial University of Newfoundland, St. John's, Canada

${ }^{3}$ Department of Medicine, University of Rochester, Rochester, USA

${ }^{4}$ School of Health Sciences, University of Ulster, Jordanstown, UK

${ }^{5}$ School of Psychology and Speech Pathology, Curtin University, Perth, Australia

${ }^{6}$ School of Sport, Exercise and Health Sciences, Loughborough University, Leicestershire, UK

${ }^{7}$ Exercise, Health and Sport Psychology, University of Western Australia, Crawley, Australia

${ }^{8}$ Nuffield Department of Orthopaedics, Rheumatology and Musculoskeletal Sciences, University of Oxford, Oxford, UK.

\section{Corresponding Author:}

Dr. Amanda Hall

Room 417 | Janeway Hostel, Health Sciences Centre

300 Prince Philip Parkway | St. John's, NL | Canada | A1B 3V6

Tel: +1 7097772958 | Fax: +1 7097776118

amanda.hall@med.mun.ca

Declaration of Interest: The authors report no declarations of interest. 


\section{Abstract (196)}

Purpose: To assess the inter-rater reliability and concurrent validity of the Communication Evaluation in Rehabilitation Tool, which aims to externally assess physiotherapists' competency in using Self-Determination Theory-based communication strategies in practice.

Materials and Methods: Audio recordings of initial consultations between 24 physiotherapists and 24 patients with chronic low back pain in four hospitals in Ireland were obtained as part of a larger randomised controlled trial. Three raters, all of whom had PhDs in psychology and expertise in motivation and physical activity, independently listened to the 24 audio recordings and completed the 18-item Communication Evaluation in Rehabilitation Tool. Inter-rater reliability between all three raters was assessed using intraclass correlation coefficients. Concurrent validity was assessed using Pearson's r correlations with a reference standard, the Health Care Climate Questionnaire.

Results: The total score for the Communication Evaluation in Rehabilitation Tool is an average of all 18 items. Total scores demonstrated good inter-rater reliability (ICC $=0.8$ ) and concurrent validity with the Health Care Climate Questionnaire total score (range: $r=0.7-$ 0.88). Item-level scores of the Communication Evaluation in Rehabilitation Tool identified five items that need improvement.

Conclusion: Results provide preliminary evidence to support future use and testing of the Communication Evaluation in Rehabilitation Tool.

Keywords: Communication; Self-determination Theory; reliability; validity;

Word Count: 3563. 


\section{Introduction}

\section{Self-determination theory and patient adherence to treatment recommendations}

Patient autonomy refers to the capacity of patients to make decisions and choices about their treatment and has been linked with improved health behaviours [1]. However, promoting patient autonomy and successfully promoting adaptive patient behaviour is challenging for many health care providers [2, 3]. Deci and Ryan's self-determination theory (SDT) [4] postulates that patients have basic psychological needs for relatedness (i.e., feeling understood and cared for), competence (i.e., feeling effective/capable in one's actions) and autonomy (i.e., feeling free to engage in the activity), which when met lead to greater autonomous patient motivation and lasting behaviour change [1, 4]. Autonomous patient motivation is defined as motivation in which the patient engages in a specific activity or behaviour because they can perceive the inherent value of it as it relates to achieving their own goals or outcomes. The use of an autonomy supportive communication style by a health care provider satisfies a patient's psychological needs and leads to increased levels of autonomous motivation, whereas a controlling communication style undermines the patient's psychological needs and leads to decreased levels of autonomous motivation for the behaviour [4]. SDT-based interventions typically include a series of communication strategies targeting a patient’s basic needs (e.g. asking open-ended questions, paraphrasing and gauging the patient's readiness to accept advice); we have previously demonstrated that this type of training can increase physiotherapists' autonomy supportive behaviour in their clinical interactions with patients [5].

\section{Patient adherence to home-based recommendations for managing chronic low back pain}

Promoting adherence to home-based recommendations such as following a series of exercises or self-management strategies is a routine part of physiotherapy or exercise-based treatment 
for patients with chronic low back pain [6]. Patient-adherence to home-based exercise as part of low back pain treatment is low [7]. To help improve patient adherence in this context, members of the author team developed an intervention based on SDT for evaluation in a fully-powered cluster randomised controlled trial (RCT), entitled the Communication Style and Exercise Compliance Trial (CONNECT) [8]. The main aim of the trial, conducted in physiotherapy settings in Dublin, Ireland, was to improve patient adherence to home-based treatment recommendations. The CONNECT intervention is an SDT-based communicationstyle training programme for physiotherapists, thus in order for it to be effective, the physiotherapists in the trial needed to adopt the communication strategies in practice with their patients. Therefore, nested within the main effectiveness RCT, we conducted an assessment of treatment fidelity; the results of this study have been published [9].

\section{Fidelity of SDT-based interventions}

While evidence suggests that SDT-based communication training interventions are effective at guiding health care providers to create supportive environments [10], less is known about how well the specific communication strategies are used by health care providers and which are most effective. Previous studies [1, 11, 12] have used the Health Care Climate Questionnaire (HCCQ) as a proxy measure of treatment fidelity [13]. While the HCCQ is designed to assess the overall level of needs support in the treatment environment, it does not provide information regarding the way in which the specific communication strategies are used by health care providers in clinical practice. This presents a problem when attempting to relate any observed changes in needs support to the specific communication strategies themselves and limits the ability to refine communication training interventions to be more effective and efficient. In an attempt to address this issue, a handful of studies [12, 14-16] assessed fidelity using the frequency of strategy implementation (i.e., the number of times 
each 'strategy' was used) in practice. However, this method is insufficient to measure competency of strategy use. For example, repeatedly using a strategy (e.g., empathising with the patient), even when it is not appropriate, may engender a feeling among patients that the health care provider's empathy is not genuine. In terms of measuring communication skill competency, there have been tools developed in other communication skills training. For example, The Behaviour Change Counselling Index has recently been developed to assesses competency of health care providers using communication techniques based on motivational interviewing [17]. Similarly, there has been research into designing a tool to assess how well physiotherapists use behaviour change techniques to promote patient self-management for those with chronic low back pain [18-19]. However, no measurement tool has been developed to specifically assess health care providers' competency in using needs supportive communication strategies in clinical practice which is also an important component for assessing fidelity of treatment delivery of SDT-based interventions [20]. Due to these issues with existing measures, in order to fully assess fidelity of the SDT-based CONNECT intervention described above, we developed a new tool, entitled, the Communication Evaluation in Rehabilitation Tool (CERT).

\section{Development of the CERT}

Item generation and scoring method: The CERT was developed as a tool to assess a physiotherapist's competency in using SDT communication strategies with patients who had low back pain. The CERT was developed in order to assess fidelity of the CONNECT intervention which was under evaluation in a larger RCT. The CERT was designed to be used by a trained rater listening to an audio/video-recording of a clinical interaction. The CERT has 18 items to align with the 18 SDT-based communication strategies used in the CONNECT intervention as listed in table 1. These strategies are common to SDT and 
therefore are generalisable to other SDT-based interventions [8]. For each strategy, one item was written to determine "how well” the physiotherapist implemented the strategy in clinical practice. Based on previous research [21, 22], a 7-point scale (1-7) was chosen, including a lower anchor (1=Not at all Well), an upper anchor ( $7=$ Very Well) and a mid-point anchor (4=Somewhat Well). To assign meaning to the scale, detailed anchor descriptions were provided [23, 24]. Face validity of the CERT scoring method was determined by reference raters in SDT and physiotherapy who pilot tested the scoring assessment using an example audio-recording of a therapist-patient encounter. The pilot test resulted in minor modifications to clarify the anchor descriptors; a copy of the final draft of the CERT can be found in supplemental material.

The CERT coder training package: The CERT is based on an observational measurement system whereby performance is rated by an objective party, thus, the generation of a coder training programme was required [25, 26]. A four-hour coder training programme was developed and included education on physiotherapy management of CLBP, SDT-based communication skills training in physiotherapy, a two-hour group training workshop on the use of the CERT, practice use of the CERT and a competency check to certify raters. The competency check consists of comparing the rater's scores on practice CERTs with ratings previously made by a reference rater using a two-way ANOVA mixed model ICC (agreement model) [27] and 95\% confidence intervals (CI). A copy of the CERT coder training manual is attached in supplemental material.

(Place table 1 near here)

Study aim: This study tests the inter-rater reliability and concurrent validity of the CERT.

\section{Materials and methods}




\section{Data Source}

The data were collected during the CONNECT trial $[8,28]$, which tested the effectiveness of an intervention designed to teach physiotherapists the 18 SDT-based communication strategies to improve their autonomy support for patients with chronic low back pain. The participants in this clinimetric study represent a subsample of the first 24 physiotherapists recruited into the CONNECT study who participated in a sub-study assessing CONNECT intervention fidelity [9]. The fidelity sub-study collected audio-recordings of the first physiotherapist-patient encounter (as part of the larger CONNECT RCT) in order to assess intervention fidelity. The methods of the fidelity sub-study are reported in full elsewhere [9], however below is a description to provide context for the current clinimetric study of the CERT.

\section{Participants}

Physiotherapists: 24 Physiotherapists (females, n=19; males, n=5) from four Dublin hospitals, all of whom were treating CLBP patients, participated in this study. Physiotherapists were aged between 28 and 46 years $(\mathrm{M}=33.71, \mathrm{SD}=4.82)$, had been practicing for between four to 22 years $(M=9.5, S D=4.4)$ and 21 held a relevant postgraduate degree. Physiotherapists provided informed written consent to participate in the CONNECT RCT as well as in the fidelity sub-study that required providingan audio-recorded session with one of their patients. Patients: 24 patients (females, $n=18$; males, $n=6$ ) referred for physiotherapy for chronic low back pain to one of the four hospitals during the recruitment period were sent an information leaflet outlining the purpose of the CONNECT RCT and fidelity study. The first author, a registered physiotherapist, screened potential participants via telephone and then in person before a physiotherapy session. Patients provided informed written consent to participate in the CONNECT RCT and the fidelity sub-study. 
Raters

The three raters who completed the training were recruited to undertake the rating for the psychometric evaluation. Three raters were recruited and completed the four-hour training programme; each of the raters had a Ph.D. in psychology with an area of expertise in motivation and physical activity [J.M., B.J., and I.T.]. The results of the training indicated ICCs (average items) between each of the raters and the reference rater ranging from 0.72 to 0.83, thus indicating good inter-rater agreement [29].

\section{Data Collection}

Ethics approval for this study was obtained from the Research Ethics Committee of each of the four participating hospitals. As described above, patients and physiotherapists provided informed written consent prior to the study commencement. A dictaphone was used to collect one audio recording of an initial appointment with a CLBP patient for each of the 24 physiotherapists. The three raters independently listened to all 24 audio files and used the CERT to rate 'how well' each of the 18 strategies were used by each physiotherapist in practice. To allow for examination of concurrent validity, raters also completed the 6-item HCCQ for each audio file, which has been established as a global indicator of perceived autonomy support [13].

\section{Reliability and validity evaluation}

In this study we assessed inter-rater reliability and concurrent validity of the CERT scores. Inter-rater reliability of CERT scores was assessed across the three independent raters. We examined reliability of the CERT total score and single item scores using a two-way mixed ANOVA, ICC (agreement model) [27] and calculated 95\% confidence intervals. Concurrent 
validity [23] was assessed used Pearson's $r$ correlations to determine the extent to which the 18-item CERT total scores (average of 18 items) correlated with the 6-item HCCQ total scores. The HCCQ assesses the degree to which a health care environment is autonomy supportive. The hypothesis was that concurrent validity of the CERT would be supported if a strong positive correlation between the CERT and HCCQ was found. Once reliability of the total scores was established, we randomly selected the CERT scores of one of the three raters and the HCCQ scores of one of the other two raters for analysis. A moderate positive correlation of $r>/=0.5$ [30] between these raters' scores was used to indicate good concurrent validity. All data were double entered and checked in MS Excel for any errors. SPSS version 21 was used for all statistical analysis [31].

\section{Results}

At an item-level, reliability was good for most items with ICCs ranging 0.5 to 0.8 indicating acceptable agreement; however, for five items the ICCs were less than 0.3 (table 2). Secondary analysis across pairs of raters showed a similar pattern of findings, suggesting agreement on these items was poor across all three raters. The ICC for the CERT total score was 0.759, suggesting overall good agreement amongst raters [30]. Pearson’s r correlation for the total 18-item scale and the HCCQ total score was $r=0.681$, demonstrating a strong positive relationship between the two variables and acceptable concurrent validity.

(Place table 2 near here)

\section{Discussion}

\section{Summary of the results}


The 18-item CERT is a theory-based tool to assess how well clinicians can use SDT-based communication strategies to support patient autonomy. This is the first study to develop such a comprehensive assessment tool and rater-training package aligned with SDT strategies to be used for promoting patient self-management with complex chronic pain conditions in rehabilitation settings. Our initial psychometric testing of the CERT provides preliminary evidence demonstrating acceptable inter-rater reliability and concurrent validity of the total score, suggesting that the CERT may be a potential tool to assess how well physiotherapists communicate in an autonomy supportive manner in clinical practice. However, while the total scores can be used as an index of needs support, there are a small number of items that warrant further refinement regarding inter-rater reliability. It is recommended that this be explored prior to widespread use.

\section{Outcome significance}

There could be several explanations for the observed low ICCs on five of the items as outlined in table 2. Firstly, many of the items obtaining high agreement among the three raters such as "goal setting” were strategies with limited opportunity for use during the consultation. Thus, it was likely easier to assess if and how well the strategy was employed. Comparatively, all of the items with low ICCs such as "using open-ended questions", “staying silent” and "paraphrasing” were strategies with a greater opportunity for use during the consultation; thus, it may be that for items with higher frequency of possible use, there was a greater opportunity for variability in determining how well they were used. Secondly, all items with low ICCs aimed to support the basic need of relatedness, as opposed to autonomy or competence; this finding is similar to those reported in a recent study of coding needs supportive behaviour in athlete coaching [32]. It has been suggested that the strategies used to code relatedness are possibly more subjective [33]. For example, relatedness is 
defined and characterised as showing care for the patient [34], which may be interpreted differently by different people. Following from this, it is possible that our coder training package did not provide enough examples of how to rate this concept more consistently from an SDT perspective. Importantly, in the SDT-based CONNECT training programme, physiotherapists were taught non-verbal aspects of communication to show relatedness. For example, not taking notes while the patient is speaking or sitting to face the patient fully when communicating with one another, were taught as good ways to demonstrate connection with and caring for the patient. Thus, in order to better rate the relatedness construct, and hopefully improve inter-rater reliability, video-recording may need to be used in future research.

Concurrent validity was assessed by examining the correlation between the scores on the CERT and scores on the HCCQ. The HCCQ is often used as the main outcome measure in SDT-based interventions to determine if the communication intervention was successful in improving the autonomy supportive environment [1]. It is, thus, also used as a proxy measure of intervention fidelity for many SDT-based communication interventions that cannot assess communication strategy use in vivo. As the SDT-based strategies are aimed directly at improving the autonomy supportive environment, the strong correlation observed in this study between the CERT and the HCCQ (6-item autonomy support subscale) provides preliminary evidence of the CERT's concurrent validity.

\section{Further testing}

There are several areas for further empirical testing with different populations and larger sample sizes. For example, based on SDT, no fixed patterns of inter-correlation among the 18 items were assumed in this study; however, this is not to say that patterns of inter-correlation 
do not exist - perhaps items that target the same psychological need of relatedness, competence or autonomy are related. It is plausible that items grouped together within the same category may be inter-correlated. We recommend that the next phase of testing of the CERT include a larger sample size with a pre-planned factor analysis to explore the interitem relationships in more detail.

Additionally, as we briefly discussed although the CERT items are largely based on verbal communication, in some cases, quality could be influenced by non-verbal cues, particularly with respect to the construct of relatedness. For example, one of the strategies, empathy, involves showing the patient that the physiotherapist understands the emotions under pinning the issue being discussed. This strategy could be influenced by where the physiotherapist is looking, either at the patient to portray engagement and interest or not looking at the patient but perhaps looking away or at the chart portraying being distracted and not interested in the treatment session. The quality of these non-verbal aspects of SDT-communication could only be assessed if direct observation or video-recording is used in further testing.

Further, the coders included in this study had backgrounds in health psychology; this limits the generalisability of our findings. To support implementation of this tool in clinical or research settings, it would be essential to further test the training package on health professionals or researchers with different educational and clinical backgrounds.

\section{Overall strengths}

The CERT assesses the quality of autonomy supportive communication, adding an extra dimension to the manner in which communication interventions can be assessed in terms of fidelity. The study aligned the development of the tool with evidence-based recommendations 
[21] and provided a detailed description of the rater-training programme for future replication. A final strength of the study was that the primary source of data was audio recordings. Direct observation methods (audio or video recordings) are deemed the 'gold standard' for this type of communication research, compared with lower quality assessments, such as checklists or post-treatment patient surveys [16].

\section{Limitations}

Although informed opinion was sought to ensure the face and content validity of items, it was a limitation that only four experts reviewed the tool. Determining an appropriate number of judges is an arbitrary process; however, face and content validity may have been enhanced further if a larger panel of expert judges, who were following a formal reviewing procedure and not involved in the development of the tool, had reviewed the initial draft of the CERT items [35]. As with observation-based outcome assessment, there is a possibility of bias via the Hawthorne effect; we aimed to minimize the potential of this bias by using a dictaphone to collect the audio recordings that was small and could easily go unnoticed by the physiotherapist during the treatment sessions. As discussed in the section on further research, our assessment method relied on audio-recordings, while this method is superior to other assessment methods such as self-report, it lacks the potential to assess non-verbal aspects of communication. Therefore, our assessment may have missed important aspects of communication that can only be assessed by direct observation, and the use of this tool would be strengthened if tested with video-recordings of treatment encounters. Lastly, the results are not generalizable to other settings. For example, in this study, the coders were all health psychologists. Therefore, it is uncertain if the same level of reliability and validity can be expected if rated by people with different professional backgrounds and training. Thus, our 
results should only be considered valid for coders with previous similar psychological training or baseline knowledge.

\section{Implications for clinical practice}

The tool was designed such that it can be adapted and used with other health care providers (e.g., general practitioners, public health nurses, dieticians, and occupational therapists) who are delivering self-management advice with a home-based component. Addressing patient adherence to self-management advice has been highlighted as a priority in health research [36]. While health care providers are best positioned to promote patient behaviour change, it is well documented that they struggle to implement behaviour change techniques [37] limiting the ultimate success of interventions $[15,17]$. Therefore, the CERT provides an opportunity to assess if health care providers are in fact using SDT-based communication strategies appropriately for promoting patient adherence to treatment recommendations.

Additionally, the CERT has a unique potential to also act as a mechanism for identifying which strategies individuals may be having difficulty implementing and providing opportunities for feedback and improvement. Absence of feedback has been highlighted by many health care providers as a barrier to successful implementation of interventions [38]. Therefore, the CERT could be used as 1) a method of providing feedback during the training process to tailor training to individual learning needs and 2) a means of identifying aspects of the intervention in which the health care provider struggled and may require further training.

Similarly, the CERT could be used during entry level training at university. Despite recommendations that training health care providers should be provided with behavioural strategies to help promote patient self-management [39], there remains an absence of 
education on the development of interpersonal communication and behaviour skills [40]. Thus, the CERT may prove a beneficial teaching aid in health care provider education.

\section{Conclusion}

This study provides the first measurement tool designed to assess the competency of SDTbased communication strategies used by physiotherapists. While further analyses of measurement properties are still warranted, the tool demonstrated good initial clinimetric properties, illustrating the potential use of the CERT in research that aims to assess appropriate use of SDT-based strategies in clinical practice.

This study was approved by the Ethics Committees at (i) Adelaide and Meath Hospital, (ii) Connolly Hospital, (iii) Beaumont Hospital and (iv) St.Vincent’s University Hospital. 
Assessing communication in rehabilitation

\section{Acknowledgements}

Funding for this study was provided by the Health Research Board (HRB) of Ireland Health Research Award 2010 (HRA_POR/2010/102), including a post-graduate scholarship awarded to the lead author. The authors thank the Dr. Chris Lonsdale for his support with development of the CERT as part of the CONNECT RCT and thank the physiotherapists and patients who participated in the project.

\section{Disclosure statement}

The authors report no conflicts of interest. 


\section{References}

1. Fortier MS, Sweet SN, O'Sullivan TL, et al. A self-determination process model of physical activity adoption in the context of a randomized controlled trial. Psychol Sport Exerc. 2007;8.

2. Cooper K, Smith BH, Hancock E. Patients' perceptions of self-management of chronic low back pain: evidence for enhancing patient education and support. Physiotherapy. 2009;95:43-50.

3. Whitlock EP, Orleans CT, Pender N, et al. Evaluating primary care behavioral counseling interventions - an evidence-based approach. Am J Prev Med. 2002;22:267-284.

4. Deci EL, Ryan RM. The general Causality Orientations Scale - Self-Determnation in Personality. J Res Pers. 1985;19:109-34.

5. Matthews J, Hall A, Hernon M, et al. A brief report on the development of a theoretically-grounded intervention to promote patient autonomy and selfmanagement of physiotherapy patients: face validity and feasibility of implementation. BMC Health Serv Res. 2015;15.

6. O’Connell N, Cook C, Wand B, et al. Clinical guidelines for low back pain: A critical review of consensus and inconsistencies across three major guidelines. Best Prac Res Clin Rheum. 2017;30:968-980.

7. Kolt GS, McEvoy JF. Adherence to rehabilitation in patients with low back pain. Man Ther. 2003;8:110-116.

8. Lonsdale C, Hall AM, Williams GC, et al. Communication style and exercise compliance in physiotherapy (CONNECT). A cluster randomized controlled trial to test a theory-based intervention to increase chronic low back pain patients' adherence to physiotherapists' recommendations: study rationale, design, and methods. BMC Musculoskel Dis. 2012;13.

9. Murray A, Hall AM, Williams G, et al. Effect of a self-determination theory-based communication skills training program on physiotherapists' psychological support for their patients with chronic low back pain: a randomized controlled trial. Arch Phys Med Rehab. 2015;96:809-16.

10. Su Y, Reeve JM. A Meta-analysis of the effectiveness of intervention programs designed to support autonomy. Educ Psychol Rev. 2010;23:159-88.

11. Williams GC, McGregor HA, Sharp D, et al. Testing a self-determination theory intervention for motivating tobacco cessation: supporting autonomy and competence in a clinical trial. Health Psychol. 2006;25:91-101.

12. Williams GC, McGregor HA, Zeldman A, et al. Testing a self-determination theory process model for promoting glycemic control through diabetes self-management. Health Psychol. 2004;23:58-66.

13. Williams GC, Lynch MF, McGregor HA, Ryan RM, Sharp D, Deci EL. Validation of the" Important Other" Climate Questionnaire: Assessing Autonomy Support for Health-Related Change. Families, Systems, \& Health. 2006;24:179

14. Alexander SC, Cox ME, Turer CLB, et al. Do the five A's work when physicians counsel about weight loss?. Fam Med. 2011;43:179-184.

15. Carroll JK, Antognoli E, Flocke SA. Evaluation of physical activity counseling in primary care using direct observation of the 5As. Ann Fam Med. 2011;9:41-22.

16. Glasgow RE, Emont S, Miller DC. Assessing delivery of the five 'As' for patientcentered counseling. Health Promot Int. 2006;21:245-55. 
17. Lane C, Huws-Thomas M, Hood K, et al. Measuring adaptations of motivational interviewing: the development and validation of the behaviour change counseling index (BECCI). Patient Educ Couns. 2005;56:166-173.

18. Harman K, MacRae M, Vallis M, et al. Working with people to make changes: a behavioural change approach used in chronic low back pain rehabilitation. Physiother Canada. 2014;66:82-90.

19. Harman K, MacRae M, Vallis M. The development and testing of a checklist to study behaviour change techniques used in treatment programme for Canadian armed forces members with chronic non-specific low back pain. Physiother Canada. 2014;66:313321.

20. Borrelli B. The assessment, monitoring, and enhancement of treatment fidelity in public health clinical trials. J Public Health Dent. 2011;71:2S52-S63.

21. DeVellis R. Scale Development: Theory and Applications. 2 ed. Vol. 26. Thousand Oaks California: Sage Publications; 2003

22. Preston CC, Colman AM. Optimal number of response categories in rating scales: reliability, validity, discriminating power, and respondent preferences. Acta Psychol. 2000;104:1-15.

23. Sim J, Wright C. Research in healthcare: concepts, designs and methods. Cheltenham: Stanley Thomas Ltd; 2000.

24. Kamper SJ, Ostelo R, Knol DL, et al. Global Perceived Effect scales provided reliable assessments of health transition in people with musculoskeletal disorders, but ratings are strongly influenced by current status. J Clin Epidemiol. 2010;63:760-66.

25. Kottner J, Gajewski BJ, Streiner DL. Guidelines for Reporting Reliability and Agreement Studies (GRRAS). Int J Nurs Stud. 2011;48:659-60.

26. Portney LG, Watkins MP. Foundations of clinical research: applications to practice. Upper Saddle River, NJ:Prentice Hall; 2000.

27. Shrout PE, Fleiss JL. Intraclass correlations - uses in assessing rater reliability. Psychol Bull. 1979;86:420-28.

28. Lonsdale C, Hall A, Murray A, et al. Communication Skills Training for Practitioners to Increase Patient Adherence to Home-based Rehabilitation for Chronic Low Back Pain : Results of a Cluster Randomised Controlled Trial. Arch Phys Med Rehab 2017, 98: DOI:10.1016/j.apmr.2017.02.025.

29. Nunnally JC, Bernstein JH. Psychometric theory. 3 ed. New York: Mcgraw-Hill; 1994.

30. Cohen J. Satistical power analysis for the behavioural sciences. 2 ed. Hillsdale, NJ: Lawerance Erlbaum Associates; 1988.

31. IBM SPSS Statistics for Windows, Version 22.0. Armonk, NY: IBM Corp.; Released 2013.

32. Smith N, Tessier D, Tzioumakis Y, et al. Development and validation of the multidimensional motivational climate observation system. J Sport Exercise Psy. 2015;37:4e22.

33. Haerens L, Aelterman N, Van den Berghe L, et al. Observing physical education teachers' need-supportive interactions in classroom setting. J Sport Exercise Psy. 2013;35:3-17.

34. Deci EL, Ryan RM. The "what" and "why" of goal pursuits: human needs and the self-determination of behavior. Psychol Inq. 2000;11:227-268.

35. Dunn J, Bouffard M, Rodgers W. Assessing item content relevance in sport psychology scale construction research: issues and recommendations. Meas Phys Educ Exerc Sci. 1999;3. 
36. Sabaté E. Adherence to long-term therapies: evidence for action. WHO; 2003.

37. Leach E, Cornwell P, Fleming J, et al. Patient centered goal-setting in a subacute rehabilitation setting. Disabil Rehabil. 2010;32:159-72.

38. Lawn S, Schoo A. Supporting self-management of chronic health conditions: common approaches. Patient Educ Couns. 2009;80:204-211.

39. Foster NE. Barriers and progress in the treatment of low back pain. BMC Med. 2011;9:108.

40. Jones LE, Hush JM. Pain education for physiotherapists: is it time for curriculum reform?. J Physiother. 2011;57:207-08. 
Table 1. CERT tool Content: SDT-based Communication strategies mapped to the construct's key components (strategies were originally developed as an intervention for clinicians in the CONNECT study, Lonsdale 2012)

\section{SDT-based Communication strategy}

Construct: autonomy support Key components according to Self-determination theory

1. Using open ended questions

Relatedness

2. Using single questions

Relatedness

\begin{tabular}{||l|l|}
\hline \hline 3. Staying silent & Relatedness \\
\hline \hline 4. Paraphrasing & Relatedness \\
\hline \hline 6. Empathizing $\quad$ Employing SMART Goal setting & Relatedness \\
\hline \hline 7. Catering for different Learning Preferences & Competence \\
\hline \hline 8. Closing the loop & Competence \\
\hline \hline 9. Gauging Patient readiness to accept advice & Autonomy \\
\hline \hline 10. Providing a rationale & Autonomy \\
\hline \hline 11. Provide opportunities for Patient Input or choice & Autonomy \\
\hline \hline 12. Autonomy supportive instead of controlling language & Autonomy and Competence \\
\hline \hline 13. Ensuring active Patient Participation in Goal setting & Autonomy and Competence \\
\hline \hline 14. Identifying barriers and obstacles & Autonomy and Competence \\
\hline \hline 15. Identifying solutions and obstacles & Autonomy and Competence \\
\hline \hline 16. Providing a rehabilitation diary & Autonomy and Competence \\
\hline \hline 17. Following-up & Autonomy and Competence \\
\hline \hline 18. Offering contact & Autonomy and Competence \\
\hline \hline
\end{tabular}

CERT: Communication Evaluation in Rehabilitation Tool; SDT: Self-determination theory; SMART: Simple, measurable, achievable, recorded, time-based. 
Table 2. ICC absolute agreement

\begin{tabular}{|c|c|c|c|c|}
\hline \multicolumn{2}{|c|}{$\begin{array}{l}\text { Question and Scale: Using the descriptions provided in the } \\
\text { coding manual, please answer the following question: } \\
\text { How well was the strategy used by the physiotherapist? }\end{array}$} & $\begin{array}{c}\text { ICC (95\% CI) } \\
(n=3)\end{array}$ & Sig & $\begin{array}{l}\text { Cron- } \\
\text { bach's } \\
\text { alpha }\end{array}$ \\
\hline $\begin{array}{c}1 \\
\text { Notat } \\
\text { Well }\end{array}$ & 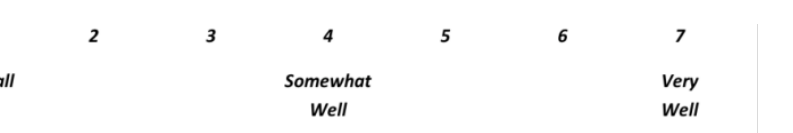 & $\begin{array}{l}\text { Type: Agreement, } \\
\text { single measures }\end{array}$ & & \\
\hline$\overline{\text { Item } 1}$ & Using open-ended questions & ". $159(-0.47-0.424)^{*}$ & 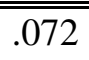 & ".397 \\
\hline Item 2 & "Using single questions & $2.214(0.007-0.471)^{*}$ & .014 & .534 \\
\hline Item 3 & "Staying silent & ".244 (0.011-0.483)* & .016 & .525 \\
\hline 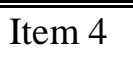 & Paraphrasing & (2.491(0.252-0.709) & .000 & ".752 \\
\hline Item 5 & Empathizing & ".628 (0.397-0.803) & 2.000 & 2.859 \\
\hline Item 6 & " Gauging patient readiness to accept advice & ".416 (0.173-0.654) & .000 & .693 \\
\hline Item 7 & Catering to different learner preferences & ".525 (0.289-0.733) & 2.000 & ".787 \\
\hline Item 8 & Closing the loop & ".673(0.356-0.848) & 2.000 & 2.911 \\
\hline Item 9 & Providing a rationale & $.487(0.239-0.708)$ & .000 & 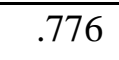 \\
\hline Item10 & Providing opportunities for patient input/choice & ".251(0.031-0.510)* & .009 & ב.559 \\
\hline Item11 & "Autonomy-supportive not controlling language & ".491 (0.250-0.709) & 2.000 & ".768 \\
\hline Item12 & $\begin{array}{l}\text { Using SMART goal setting } \\
\end{array}$ & $7.704(0.402-0.865)$ & .000 & .921 \\
\hline Item13 & "Ensuring active participation in goal setting & $7.785(0.627-0.892)$ & .000 & .922 \\
\hline Item14 & Identifying barriers and obstacles & 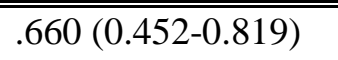 & 2.000 & ב.862 \\
\hline Item15 & $\begin{array}{l}\text { Identifying solutions and enablers } \\
\end{array}$ & ". $191(-0.051-0.486)^{*}$ & .000 & 2.425 \\
\hline Item16 & Providing a rehabilitation diary & ".712 (0.516-0.853) & 2.000 & ".877 \\
\hline Item17 & Following-up & $.492(0.251-0.711)$ & .000 & .747 \\
\hline Item18 & Offering contact & .622 (0.401-0.797) & 2.000 & 8.827 \\
\hline Total & & "759 (0.548-0.889) & .000 & .921 \\
\hline
\end{tabular}

* The ICC is below the threshold for "good" agreement. ICC: Intraclass correlation coefficient; Sig: significance; SMART: Simple, measurable, achievable, recorded, time-based. 
Assessing communication in rehabilitation 